\title{
QUANTITATIVE ANALYSIS OF CARBON ISOTOPE RATIOS IN N-, SC-, AND J-TYPE CARBON STARS
}

\author{
K. OHNAKA* AND T. TSUJI \\ Institute of Astronomy, The University of Tokyo \\ Mitaka, Tokyo, 181 Japan \\ * Now at Technische Universität Berlin, Germany
}

\begin{abstract}
We present the results of a quantitative analysis of ${ }^{12} \mathrm{C} /{ }^{13} \mathrm{C}$ ratios in $62 \mathrm{~N}$-type, $15 \mathrm{SC}$-type, and $26 \mathrm{~J}$-type carbon stars. The ${ }^{12} \mathrm{C} /{ }^{13} \mathrm{C}$ ratios are determined from lines of the CN Red System around $8000 \AA$, based on the iso-intensity method and line-blanketed model atmospheres. The resulting ${ }^{12} \mathrm{C} /{ }^{13} \mathrm{C}$ ratios in $\mathrm{N}$ - and $\mathrm{SC}$-type carbon stars are consistent with a scenario in which $\mathrm{M}$ giants evolve through $\mathrm{SC}$ - to $\mathrm{N}$-type carbon stars, as ${ }^{12} \mathrm{C}$ produced during thermal pulses is added to the envelope.
\end{abstract}

\section{Introduction}

The carbon isotope ratio brings us important information about the nucleosynthesis and mixing in the stellar interior, because carbon is one of the elements involved in the nucleosynthesis during thermal pulses and mixing to the surface. If the ${ }^{12} \mathrm{C}$ synthesized in thermal pulses is responsible for the formation of carbon stars, then ${ }^{12} \mathrm{C} /{ }^{13} \mathrm{C}$ ratios are expected to be enhanced as compared with those of their progenitors, and to have a correlation with $\mathrm{C} / \mathrm{O}$ ratios.

To demonstrate this correlation, we have carried out a quantitative analysis of carbon isotope ratios from lines of $\mathrm{CN}$ located around $8000 \AA$ for a large sample of carbon stars, including $\mathrm{N}, \mathrm{SC}$, and $\mathrm{J}$ stars. Our main purpose is to determine the distribution of ${ }^{12} \mathrm{C} /{ }^{13} \mathrm{C}$ ratios of each type of carbon star based on a large sample, and to find some connections between carbon stars and $\mathrm{K}$ and $\mathrm{M}$ giants, which are considered to be their progenitors. 


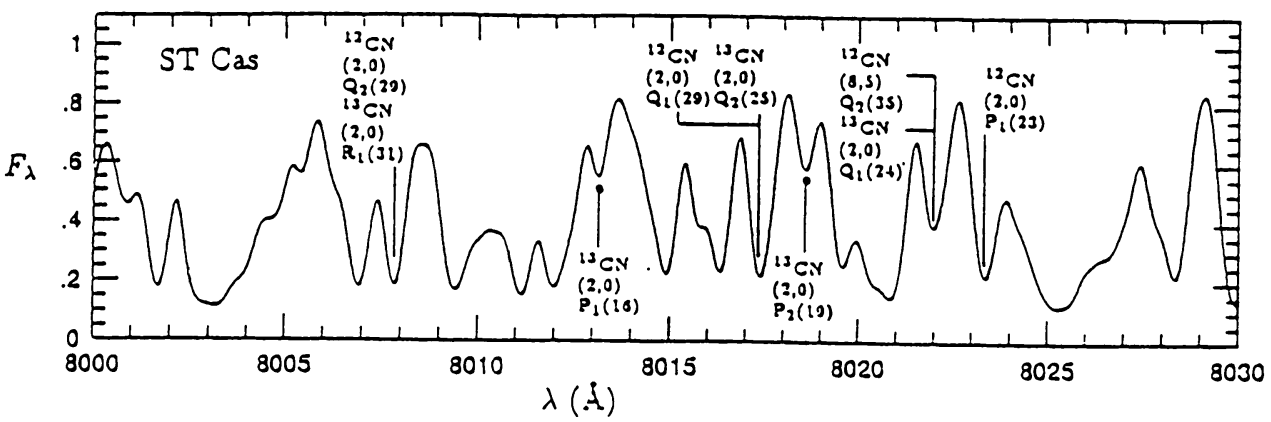

Figure 1. Observed spectrum of an N-type carbon star, ST Cas.

\section{Observations}

All observations were carried out using the 74-inch telescope of Okayama Astrophysical Observatory $\left(\mathrm{OAO}^{1}\right)$, during the period between October 1987 and March 1994. Our sample consists of $66 \mathrm{~N}$-type, 19 SC-type, and $26 \mathrm{~J}$-type carbon stars. Lists of our program stars are given in Ohnaka \& Tsuji $(1996,1998)$. All spectra consist of two adjacent exposures, covering from 7800 to $8030 \AA$. Each exposure covers $120 \AA$ with an overlap of $10 \AA$. The spectral resolution is about 20,000. The signal-to-noise ratios are about 100 , although somewhat lower for faint stars and much higher for some of the brightest stars.

\section{Analysis}

The observed region is very crowded with lines due to the Red System of ${ }^{12} \mathrm{CN}$ and ${ }^{13} \mathrm{CN}$ and the Phillips System of $\mathrm{C}_{2}$, as can be seen in Figure 1. We selected about 10 lines of ${ }^{12} \mathrm{CN}$ and 2 lines of ${ }^{13} \mathrm{CN}$ for each star. Some lines are blended with other lines at almost the same wavelength. We used them in the analysis only if the blending line is weak enough compared to the line in question, considering its $g f$-value and lower excitation potential. Central depths are normalized by the fictitious continuum level, which is drawn to go through the highest point in the observed region and is assumed to be constant over the region.

We adopted the iso-intensity method for the analysis. It is similar to the curve-of-growth method, but we use the central depths of lines as the ordinate, instead of equivalent widths. For lines of ${ }^{12} \mathrm{CN}$ and ${ }^{13} \mathrm{CN}$, logarithms of the observed central depths normalized by the fictitious continuum level are plotted against $\log (g f \Gamma)$, which is the line intensity predicted by the weighting-function method (e.g. Cayrel \& Jugaku 1963). The horizontal

${ }^{1} \mathrm{OAO}$ is a branch of the National Astronomical Observatory of Japan (NAOJ). This work was carried out under the common use program of OAO. 

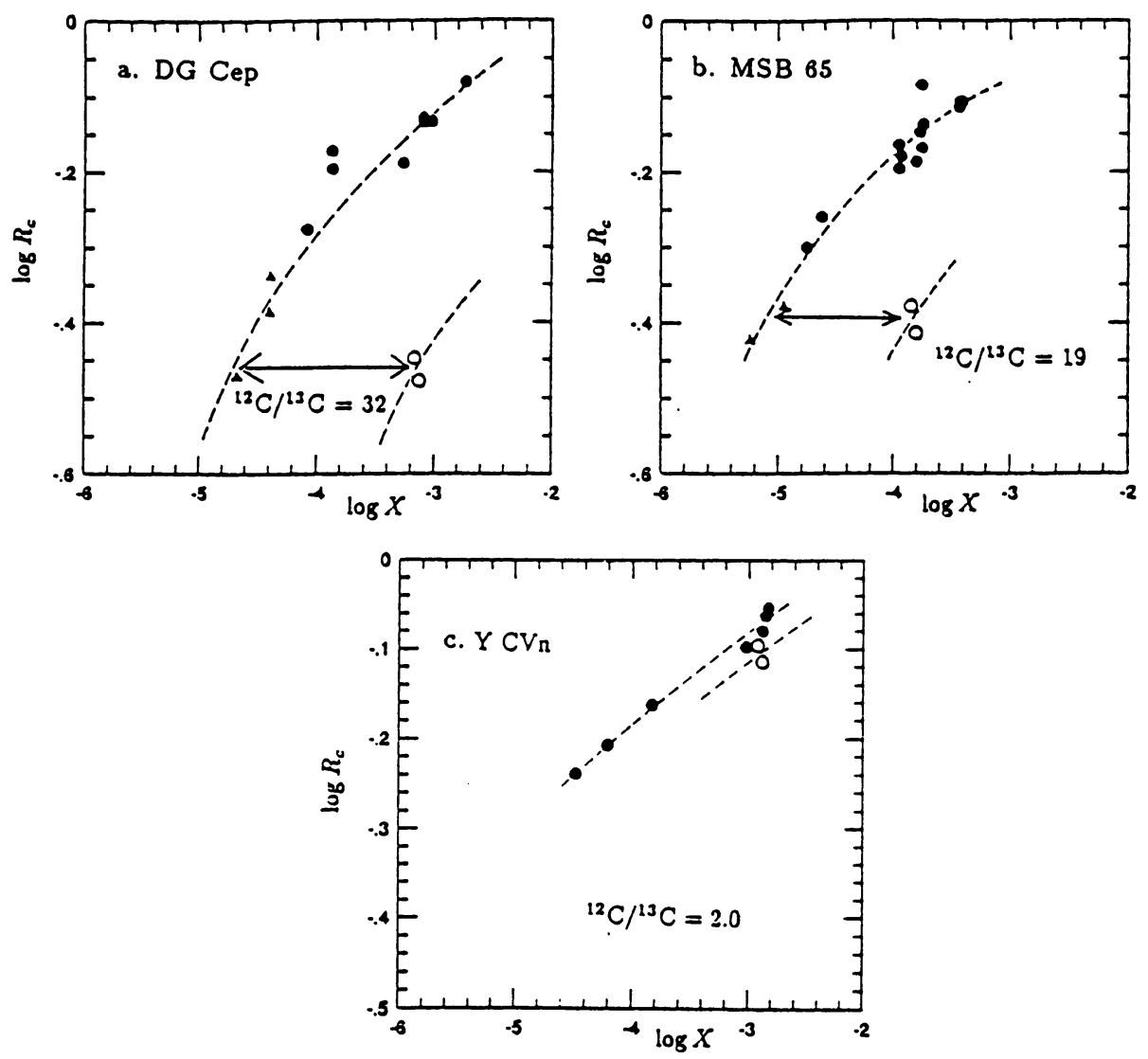

Figure 2. Examples of curves-of-growth: (a) DG Cep; (b) MSB 65; (c) Y CVn.

shift between the two curves gives the isotopic ratio. Examples are shown in Figure 2.

It should be noted that the horizontal shift between the curves for ${ }^{12} \mathrm{CN}$ and ${ }^{13} \mathrm{CN}$ is measured at the same depth; hence, the continuum location does not affect the resulting isotopic ratio as long as the continuum is constant in the observed region. Since the carbon isotope ratios are determined by lines of ${ }^{12} \mathrm{CN}$ and ${ }^{13} \mathrm{CN}$ of the same intensity, what really matters is that the central depths normalized by the fictitious continuum are a kind of measure of line intensity which serves to recognize that the lines of ${ }^{12} \mathrm{CN}$ and ${ }^{13} \mathrm{CN}$ are iso-intensity.

\section{Results}

The distributions of the derived ${ }^{12} \mathrm{C} /{ }^{13} \mathrm{C}$ ratios of each type of carbon star are shown in Figure 3. The average ${ }^{12} \mathrm{C} /{ }^{13} \mathrm{C}$ ratio is $27 \pm 11$ (standard 
deviation) for $\mathrm{N}$-type carbon stars and $22 \pm 14$ for SC-type carbon stars. The average ${ }^{12} \mathrm{C} /{ }^{13} \mathrm{C}$ ratio in the $26 \mathrm{~J}$-type carbon stars is $4.7 \pm 2.8$. The ${ }^{12} \mathrm{C} /{ }^{13} \mathrm{C}$ ratios found for each star are given in Ohnaka \& Tsuji (1996, 1998).

Figure $3 a$ clearly shows that the most frequent ${ }^{12} \mathrm{C} /{ }^{13} \mathrm{C}$ ratios among $62 \mathrm{~N}$ stars are between 20 and 30 . Nearly half of our program stars have ratios in this range. This is in marked contrast with the result of Lambert et al. (1986) who reported that the most frequent ratios among the 24 $\mathrm{N}$ stars they studied are from 50 to 60 . As one possible reason for this disagreement, we noticed that their model atmospheres may be cooler by more than $500 \mathrm{~K}$ in the line-forming region than our models of the same effective temperature. However, this difference in the model atmospheres may be due to the inclusion of turbulent pressure in our model atmospheres, since the comparison of the models was done on the $\log P_{g}-T$ plane. Thorough tests of the model atmospheres should be done in order to resolve this discrepancy.

\section{Discussion}

\subsection{N AND SC STARS}

We now discuss how an oxygen-rich star evolves into a carbon star, in terms of chemical compositions. We consider the scenario in which an $\mathrm{M}$ giant evolves to an $\mathrm{N}$ type carbon star as ${ }^{12} \mathrm{C}$ produced in the helium shell flash is dredged up to the surface, and SC stars are transitional objects between oxygen-rich stars and carbon stars. First, analyses of chemical compositions of $\mathrm{M}$ giants were done by Smith \& Lambert $(1985,1990)$, who showed that $\mathrm{C} / \mathrm{O}=1 / 2-1 / 3$ and ${ }^{12} \mathrm{C} /{ }^{13} \mathrm{C}=10-20$ in $\mathrm{M}$ giants. On the other hand, $\mathrm{C} / \mathrm{O}$ ratios in $\mathrm{N}$ stars have been analyzed by Lambert et al. (1986), who found that the average $\mathrm{C} / \mathrm{O}$ ratio in $24 \mathrm{~N}$ stars is 1.1. In other words, if we assume that the oxygen abundance is not modified by the third dredge-up, the carbon abundance is doubled or tripled by the addition of ${ }^{12} \mathrm{C}$ in the progression from an $\mathrm{M}$ giant to an $\mathrm{N}$ star. Therefore, the ${ }^{12} \mathrm{C} /{ }^{13} \mathrm{C}$ ratio should also be doubled or tripled from 10 up to $\sim 20$ or 30 , which is consistent with our result for $\mathrm{N}$ stars. Besides, $\mathrm{C} / \mathrm{O}$ ratios of $\mathrm{SC}$ stars are considered to be nearer to unity, that is, less than the value 1.1 of $\mathrm{N}$ stars. This means that the amount of ${ }^{12} \mathrm{C}$ added to the surface of SC stars is almost equal to that added to the surface of $\mathrm{N}$ stars. This implies that the ${ }^{12} \mathrm{C} /{ }^{13} \mathrm{C}$ ratios of SC stars should have a distribution similar to that of $\mathrm{N}$ stars. Our results (Figs. $3 a$ and $3 b$ ) show that the range of the ${ }^{12} \mathrm{C} /{ }^{13} \mathrm{C}$ ratios of $\mathrm{SC}$ stars is not so different from that of the ${ }^{12} \mathrm{C} /{ }^{13} \mathrm{C}$ ratios of $\mathrm{N}$ stars. 

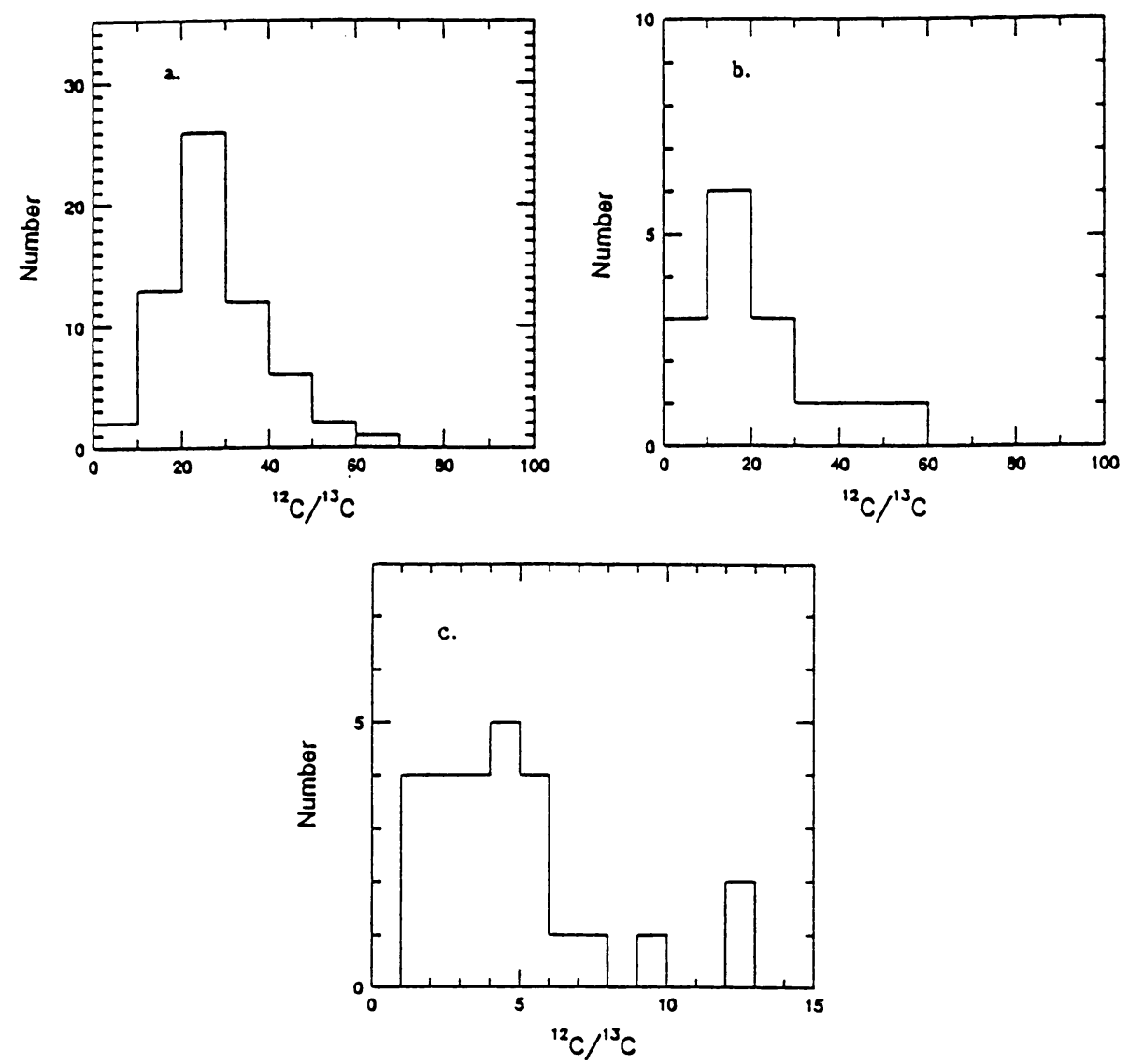

Figure 3. Histograms of the ${ }^{12} \mathrm{C} /{ }^{13} \mathrm{C}$ ratios obtained for (a) N-type, (b) SC-type, and (c) J-type stars.

\subsection{J STARS}

In Figure 4, we plot the galactic latitude $|b|$ of each $\mathrm{J}$ star against the derived ${ }^{12} \mathrm{C} /{ }^{13} \mathrm{C}$ ratio. Though the plot appears to be rather scattered, it seems that the $\mathrm{J}$ stars with relatively large ${ }^{12} \mathrm{C} /{ }^{13} \mathrm{C}$ ratios tend to be at low galactic latitudes whereas the extremely ${ }^{13} \mathrm{C}$-rich $\mathrm{J}$ stars are distributed at all galactic latitudes. However, since the number of high-latitude stars observed on our program is relatively small, it would be desirable to analyze more stars at high galactic latitudes to confirm the above correlation.

We now turn to the silicate carbon stars in our sample. The ${ }^{12} \mathrm{C} /{ }^{13} \mathrm{C}$ ratios are $5.3 \pm 0.9$ for EU And, $4.8 \pm 0.8$ for BM Gem, $4.8 \pm 0.8$ for V 778 Cyg, $3.0 \pm 1.0$ for NC 83, and 5.6 \pm 1.4 for GCCCS 447 . These results are perfectly consistent with the previous identification of these stars as J-type. And it is worth noting that the five silicate carbon stars have ${ }^{12} \mathrm{C} /{ }^{13} \mathrm{C}$ ratios 


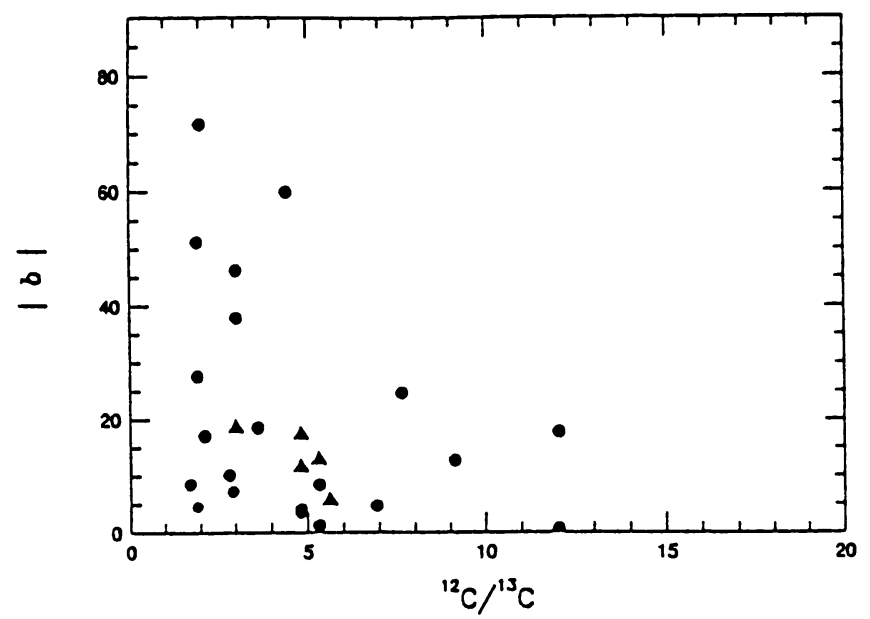

Figure 4. Galactic latitude $|b|$ of each $\mathrm{J}$ star is plotted against its ${ }^{12} \mathrm{C} /{ }^{13} \mathrm{C}$ ratio. Filled triangles represent the silicate carbon stars.

which are among the most common values in our sample. In other words, these stars exhibit no peculiar ${ }^{12} \mathrm{C} /{ }^{13} \mathrm{C}$ ratios which would be associated with the presence of the silicate emission feature.

\section{Future Work}

To clarify the complicated evolution of carbon stars and related objects, further analyses of the elemental abundances of $\mathrm{C}, \mathrm{N}, \mathrm{O}$, and their isotopes are indispensable. In addition, determinations of the abundances of the $s$-process elements are highly desirable. We should also investigate the mechanism of mass loss, which no one doubts plays an important role in stellar evolution at the final stage. The Infrared Space Observatory (ISO) will bring us spectra of objects at the final stage of stellar evolution. These spectra will help to clarify the physics and chemistry of circumstellar shells. This kind of approach is expected to show how mass loss during the evolution from the AGB to planetary nebulae is related to the formation of carbon stars and related objects.

\section{References}

Cayrel, R. \& Jugaku, J. 1963, Ann d'Astrophys, 26, 495

Lambert, D. L., Gustafsson, B., Eriksson, K. \& Hinkle, K. H. 1986, ApJS, 62, 373

Ohnaka, K. \& Tsuji, T. 1996, $A \& A, 310,933$

Ohnaka, K. \& Tsuji, T. 1998, $A \& A$, submitted

Smith, V. V. \& Lambert, D. L. 1985, $A p J, 294,326$

Smith, V. V. \& Lambert, D. L. 1990, ApJS, 72, 387 


\section{Discussion}

Little-Marenin: Some J-type stars are relatively close to us (e.g. Y CVn), and even though they are at high galactic latitude, their distance above the galactic plane is fairly small. Will this affect the ${ }^{12} \mathrm{C} /{ }^{13} \mathrm{C}$ - latitude effect that you found? Did you consider this?

Ohnaka: Distances for $\mathrm{C}$ stars are difficult to determine; I will consider this in more detail.

Gustafsson: The difference in ${ }^{12} \mathrm{C} /{ }^{13} \mathrm{C}$ between Ohnaka \& Tsuji and Lambert et al. (1986) is not easy to explain. The methods are different - Lambert et al. used $\Delta v=2 \mathrm{CO}$ lines and CN Red System lines in the $2.2 \mu \mathrm{m}$ band, and there we claim that the continuum can be traced, while you rely on comparing lines with similar depths without knowing the level of the continuum. Your models have lower gas pressures than ours (basically because you include a turbulent pressure) but I doubt whether that matters. Our temperature scale is about $200 \mathrm{~K}$ lower than yours, but that would only - at the most - explain half of the effect. The reason for the discrepancy and the model sensitivity of the two methods should be further explored.

Ohnaka: First, the models should be compared in the $\tau-T$ plane. That comparison will tell us more exactly how different the two models are. Second, a re-analysis of the spectra that Lambert et al. (1986) analyzed should be done with our model atmospheres. This should serve to explore the reasons for the discrepancies in the ${ }^{12} \mathrm{C} /{ }^{13} \mathrm{C}$ ratios. 